\title{
PYELOGRAPHY IN INFANTS
}

\author{
BY
}

W. E. UNDERWOOD, F.R.C.S.,

Chief Assistant to a Surgical Unit, St. Bartholomew's Hospital.

Pyelography in infants is an examination which is essential under certain circumstances, and from it valuable facts may often be obtained which would be undiscovered without this specialized form of investigation. Hitherto the examination has been surrounded by difficulties of such a nature that it is often unsuccessful and the child is submitted to discomfort without result. The object of this paper is to bring forward certain notes on cases where pyelography has been indicated. The observations from a series of sixteen cases have led to the development of a method whereby good pyelograms have been obtained with certainty.

Instrumental pyelography in infancy is a procedure not to be advised lightheartedly, but there are occasions where the indications are definite and adequate: in these cases the anticipation of possible information to be gained justifies submitting the infants to what constitutes a major examination. In this series are cases of urinary infection resistant to the usual medical treatment, of renal pain, of renal calculi, and cases of congenital malformation of the urinary tract similar to those described by Poynton and Sheldon ${ }^{1}$. The term pyelography is used here for brevity rather than accuracy, for it embraces a complete investigation of the urinary tract, including ureterography.

Methods of pyelography.-The choice lies between intravenous and retrograde or instrumental methods. After consideration of these cases it appears clear that an instrumental pyelogram is preferable for many reasons. In an intravenous pyelogram the shadows obtained are not dense and they may become invisible after the slightest movement of the kidneys with respiration. The interpretation of the intravenous pyelogram is sometimes fallacious, failing to show pathological conditions which are eventually clearly demonstrated by an instrumental pyelogram. In certain cases of congenital deformity of the urinary tract, such as hydro-ureter and hydro-nephrosis, an intravenous pyelogram fails to give any shadow until six hours after injection of the uroselectan and even then quite frequently no shadow is seen. The difficulties of radiography in children are familiar to all and it is clear that a method taking at least three-quarters of an hour is undesirable.

Anaesthesia.-When the instrumental method is employed anaesthesia is essential. Even when instruments cannot be used, as for example in the 


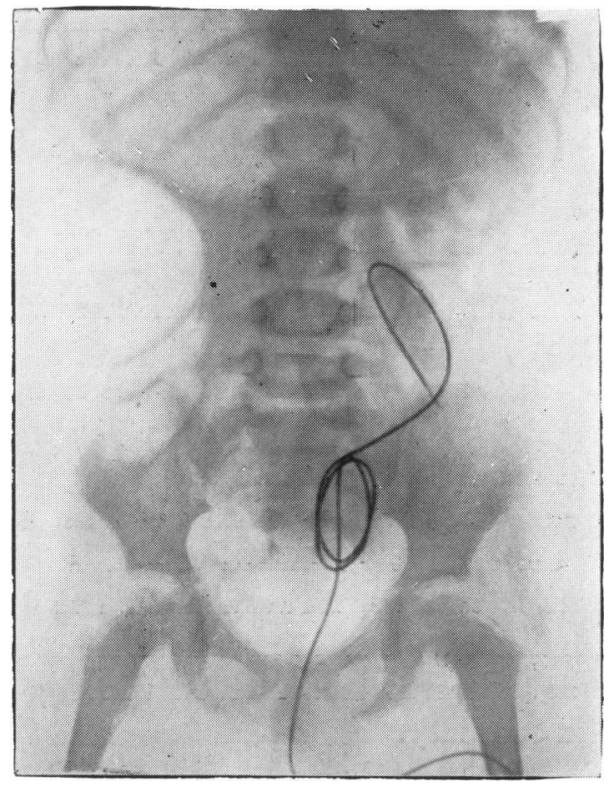

Fig. 1.

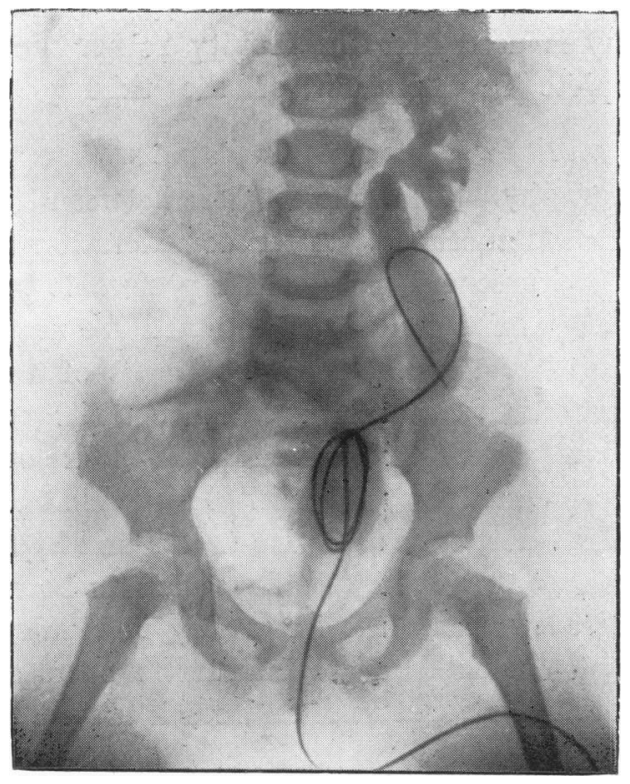

FIG. 2. 
case of a male infant with a small external urinary meatus, it is generally preferable to give an anaesthetic for the following reasons:-

(1) Without an anaesthetic it is possible to inject the uroselectan into a vein but the subsequent mental and physical upset results in a blurred pyelogram however short the exposure.

(2) By giving an anaesthetic a satisfactory still pyelogram may be obtained by a method to be described later.

The choice of anaesthetic is important: nitrous oxide and oxygen anaesthesia is adequate and safe, there are no unpleasant after-effects and it can be made to play an important part in the radiographic technique.

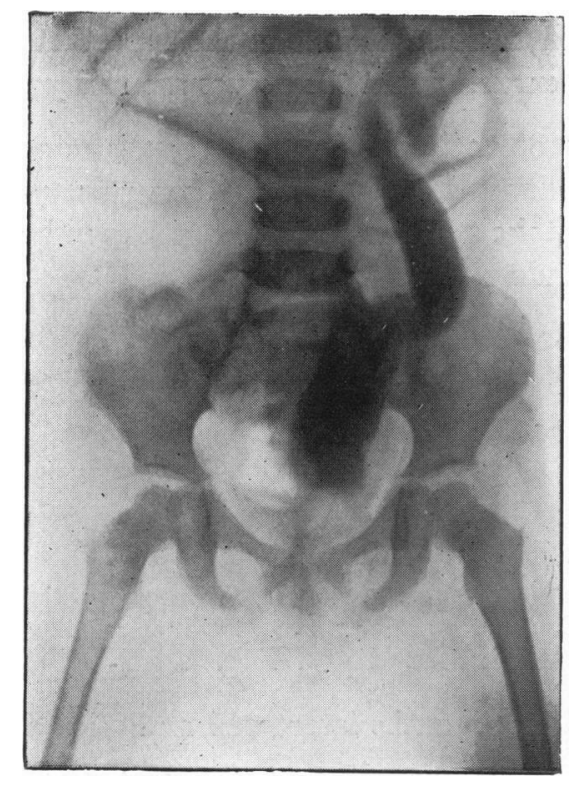

Fig. 3.

Radiographic difficulties have been discussed by Beer and Hyman ${ }^{2}$ who state that the youngest child on whom they have carried out double pyelography was two years old. They also say, 'Owing to the movement of the infant, all x-ray exposures must be instantaneous and without a Buckey diaphragm at the best it is difficult to obtain perfect pictures with regularity.' Exposures from half a second upwards are desirable for detail in pyelography using a Buckey diaphragm or a Lysholm grid, otherwise the shorter, so-called instantaneous, exposures of one-tenth of a second must be used which give neither good definition nor, for certainty, still pyelograms.

Instrumental pyelography.-The following method, if well-trained assistants are available, enables the pyelography to be carried out in a very 
short time. The cystoscope is passed and the interior of the bladder examined; very little time should be spent on this unless some gross abnormality is seen, in which case the knowledge gained by longer examination will repay the time spent. When the ureter or ureters have been catheterised with No. 3 catheters, the bladder is emptied and the cystoscope withdrawn; ureteric catheter specimens of urine may now be collected if this is necessary. After tilting the infant 20 degrees into a Trendelenberg position, the renal regions are examined under the screen to verify the position of the ureteric catheters; the renal pelves are then filled with 20 per cent. sodium bromide solution. It is important that this filling of the renal pelves should be controlled by sight, under the screen, for these reasons:-

(1) The usual way of verifying that the pelves are filled, by the patient experiencing renal pain, is not available in the anaesthetized infant and vision is the only method of ensuring that the pelves are really full.

(2) The capacity of an infant's renal pelvis is normally 2 or 3 c.c. and it would be unwise blindly to inject more than this, yet if an abnormality exists the pelvis may take 5,10 or even 15 c.c. before being sufficiently filled to give the correct shadow.

It is impossible to obtain true pyelograms without risk of over-distension, unless this be done by direct vision under the screen.

The three uretero-pyelograms Fig. 1, 2 and 3 illustrate this point. They were taken after 5 c.c., 10 c.c. and finally a total of 15 c.c. had been injected on the right side. In this case the opposite pelvis was normal, holding only 2.5 c.c. It was not until the total 15 c.c. had been injected and the ureteric catheter withdrawn that the pelvis and ureter on the right were completely filled and an accurate uretero-pyelogram obtained. The kidney and ureter were infected, nephrectomy and excision of the ureter were performed and the child left hospital two months later, well and with a sterile urine.

Immediately the pelves are filled, the anaesthetic is discontinued and after approximately a minute an x-ray is taken. The infant is placed in the reversed Trendelenberg position, the ureteric catheters are withdrawn while injecting a further 1 c.c. of bromide solution and a skiagram of the filled ureters and renal pelves taken.

The whole difficulty in taking these pyelograms is to secure a period of inhibited respiration sufficiently long to enable the radiographer to make an adequate exposure and it has already been shown that not less than half a second is needed. Previously an exposure of one-fifth of a second was made during quiet respiration in the hope that a still picture would result, and blurred pyelograms were only too familiar.

Fig. 4, 5, 3 represent the experimental record of a pyelography on a child two years old. The respiratory movements were obtained from a rubber bag lightly strapped over the infant's chest and connected with a tambour. The lowest tracing represents a time interval in half seconds; the intermediate 
line marks the beginning and end of the x-ray exposure. Fig. 4 shows a tracing of quiet shallow respiration under the anaesthetic. It will be seen from this chart that there is insufficient time at any part of the curve to

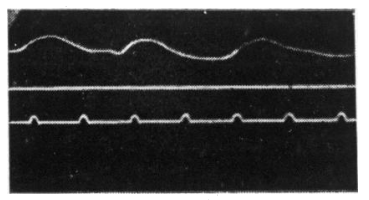

Fig. 4.
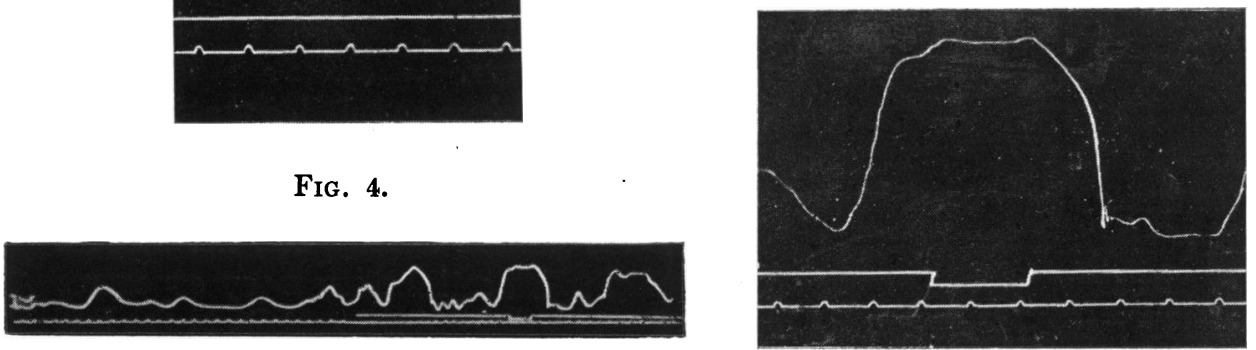

$\uparrow$

Fig. 5.

$\uparrow$

FIG. 6.

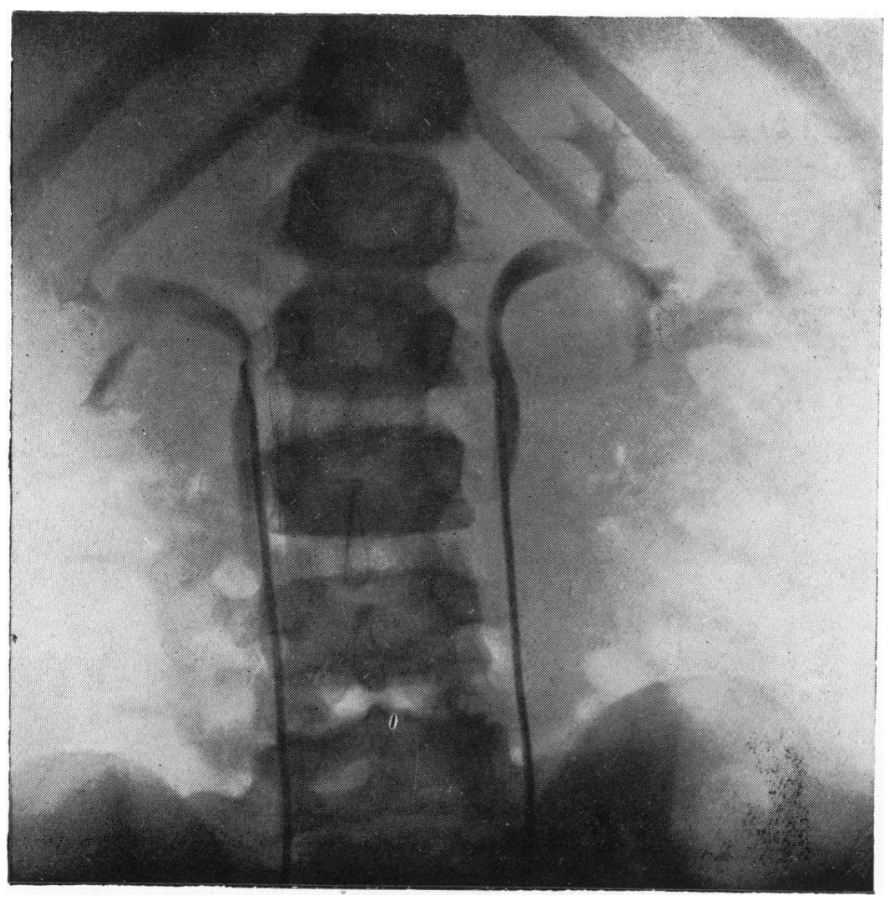

Fig. 7.

allow an adequate exposure with any chance of obtaining a still pyelogram. Fig. 5 shows the whole series of respirations from the moment the anaesthetic was discontinued at the point $\mathrm{X}$ to the point $\mathrm{Y}$ where a successful pyelogram

Note.-In Fig. 5 the tracing on the smoke drum has been broadened for clearness of reproduction, the other tracings were photographed direct from the drum. 
had been obtained. The time X Y varies with different infants, the length of anaesthesia and other factors but is in the region of one minute, sometimes being as long as three. It will be seen that there are periods of apnoea followed by intermittent deep inspirations, the inspiration $\mathbf{Y}$ being deeper and more sustained, the tracing showing a typical plateau. Fig. 6 shows the point $Y$ on a larger scale where an $x$-ray exposure of one second was possible during which the diaphragm was absolutely at rest, thus allowing a still and consequently clearly defined pyelogram.

The periods of apnoea often suggest themselves as suitable moments for $\mathrm{x}$-ray exposure, but they are found to be of variable length and the next inspiration may occur unexpectedly. On the other hand, once there occurs the sustained inspiration, which gives the plateau tracing, one can be sure of subsequent similar inspirations during which satisfactory x-ray exposures can be made. This method has now been carried out successfully in the last twelve consecutive cases.

The actual skiagram taken while the observations shown in Fig. 6 were made, appears as Fig. 7.

I am indebted to Dr. Charles Harris for permission to publish some of this material and to Mr. Girling Ball for allowing me to investigate the cases and record the observations.

\section{REFERENGES.}

1. Poynton, F. J., \& Sheldon, W. P. H., Arch. Dis. Childh., London, 1927, II, 251.

2. Beer, E., \& Hyman, A., Diseases of the Urinary Tract in Children, New York, 1930, 12. 